\title{
Cotidiano, esquizofrenia e narrativas da experiência de adoecimento ${ }^{1}$
}

\author{
Ellen Cristina Ricci, Erotildes Maria Leal \\ Departamento de Saúde Coletiva, Faculdade de Ciências Médicas, Universidade Estadual de Campinas - \\ UNICAMP, Campinas, SP, Brasil.
}

\begin{abstract}
Resumo: Introdução: Apresenta-se uma revisão bibliográfica narrativa de estudos sobre o cotidiano de pessoas com diagnóstico do espectro esquizofrênico a partir das suas narrativas sobre a experiência do adoecimento, publicados na forma de artigos em revistas indexadas. As revisões narrativas partem de questões amplas, com fontes de dados e seleção de artigos que podem conter algum viés, buscando o desenvolvimento de um tema de forma contextual e teórica. Objetivo: O objetivo foi indicar como os estudos de narrativas sobre o cotidiano e experiências de adoecimento em esquizofrenia apresentam-se no cenário nacional e internacional, os autores mais relevantes, como conceituam cotidiano, tipos de estudos realizados e as possíveis contribuições para processo saúde/doença/cuidado na assistência em saúde mental. Método: Buscou-se a amplitude do material pesquisado, a apropriação e o fichamento deste. Relatamos o que foi encontrado, em termos quantitativos sobre o tema, para em seguida apresentar a análise geral dos artigos selecionados. Neste trabalho nos interessou conhecer aqueles que apresentam como o cotidiano é experimentado por pessoas com o diagnóstico de esquizofrenia. Resultados: Considerando as sete bases de dados utilizadas durante esta revisão, selecionamos 281 artigos, $90 \%$ destes de origem internacional e pouco menos de um terço (82 artigos) relatam/descrevem e valorizam as narrativas em primeira pessoa sobre a experiência de adoecimento. Conclusão: Discute-se a pertinência e responsabilidade das pesquisas em saúde mental centradas no vivido, no cenário contemporâneo das ciências, e o diálogo com as singularidades e respeito às diferentes experiências de adoecimento no contexto sociocultural brasileiro.
\end{abstract}

Palavras-chave: Cotidiano, Narrativas, Experiência de Adoecimento, Esquizofrenia, Revisão Bibliográfica.

\section{Everyday life, schizophrenia and narratives of illness experience}

\begin{abstract}
Introduction: This paper presents a narrative review of the literature on the everyday life of people diagnosed with the schizophrenia spectrum, from their narratives about the illness experience, published as articles in indexed journals. The narrative reviews start from broad issues with data sources and selection of articles that may contain some bias, seeking to develop a contextual and theoretical theme. Objective: The main objective is to indicate how narrative studies on the everyday life and experience of schizophrenia are presented in the national and international scenario; the most relevant authors; how the everyday life concept is described; type of studies performed and the possible contributions to the health/disease/care in mental health care process. Method: We sought the breadth of the researched material, appropriation and organization of it. We reported the findings in quantitative terms on the subject to then present an overview of the selected papers. We aimed to knowing those who present the everyday life experienced by people diagnosed with schizophrenia. Results: Considering the seven databases used during this review, we selected 281 papers, $90 \%$ of them were international and just under one third (82 papers) report/describe and value their narrative in first person about the illness experience. Conclusão: We discuss the relevance and responsibility of mental health researches centered on the experience, the current sciences scenario, and the dialogues with singularities, and regarding the different experiences of illness in the Brazilian sociocultural context
\end{abstract}

Keywords: Everyday Life, Narratives, Illness Experience, Schizophrenia, Review Literature.

Autor para correspondência: Ellen Cristina Ricci, Laboratório Saúde Coletiva e Saúde Mental, Universidade Estadual de Campinas Rua Tessália Vieira de Camargo, 126, Cidade Universitária Zeferino Vaz, CEP 13083-887, Campinas, SP, Brasil, e-mail: ellenricci@gmail.com Recebido em Nov. 30, 2014; $1^{\text {a }}$ Revisão em Mar. 31, 2015; $2^{\text {a }}$ Revisão em Jul. 27, 2015; $3^{\text {a }}$ Revisão em Nov. 24, 2015; Aceito em Dez. 21 , 2015. 


\section{Introdução}

Este artigo apresenta a revisão bibliográfica narrativa realizada para subsidiar a discussão principal do projeto de mestrado intitulado: "Cotidiano e Esquizofrenia: narrativas de usuários de Centros de Atenção Psicossocial (CAPS) a partir da experiência de adoecimento", que teve por objetivo principal conhecer o cotidiano de usuários com diagnóstico do espectro esquizofrênico inseridos em CAPS a partir das suas experiências de adoecimento.

As revisões narrativas partem de questôes amplas, com fontes de dados e seleção de artigos que podem conter algum viés, mas o seu foco principal é buscar o "estado da arte" ou o desenvolvimento de um tema de forma contextual e teórica através de análise crítica (ROTHER, 2007).

Os passos desta revisão seguiram o que Minayo (2012) propóe em relação à amplitude do material pesquisado, apropriação e fichamento deste. Relatamos o que foi encontrado, em termos quantitativos sobre o tema, para em seguida apresentar análise geral dos artigos selecionados. O objetivo foi indicar como os estudos de narrativas sobre o cotidiano e experiências de adoecimento em esquizofrenia apresentam-se no cenário nacional e internacional, os autores mais relevantes, como conceituam cotidiano, tipos de estudos realizados e as possíveis contribuiçôes para processo saúde/doença/cuidado na assistência em saúde mental.

Os estudos sobre esquizofrenia são muitos. Neste trabalho nos interessou conhecer aqueles que apresentam como o cotidiano é experimentado por pessoas com o diagnóstico de esquizofrenia.

Estudos publicados na forma de artigos em revistas indexadas, que utilizam o conceito de cotidiano, mesmo no campo da terapia ocupacional, em que o conceito é central nas práticas, são escassos. Salles e Matsukura (2013) encontram 12 artigos no período de 2003 a 2012, embora observem crescimento na produçáo dos estudos do conceito de cotidiano. Dantas e Oda (2014), em revisão bibliográfica sobre estudos de avaliação sobre serviços de saúde mental oferecidos à população brasileira, afirmam que a minoria destes valoriza ou coloca em foco a voz dos usuários sobre a atenção prestada.

Este artigo indica que no campo científico, entendido como o estado das relações de força entre os protagonistas em luta, agentes ou instituiçóes e de distribuição desigual (BOURDIEU, 1983), a luta aparece de forma desigual entre os estudos (estudos pautados no modelo biomédico e estudos narrativos pautados na experiência do adoecimento e o cotidiano), visto que os resultados quantitativos diminutos dos estudos narrativos com foco na experiência das pessoas continuam em número menor que os estudos em que avaliam o cotidiano da perspectiva do observador externo e náo de quem o vivencia. Apresenta-se a seguir a revisão narrativa de estudos sobre as narrativas de experiência de adoecimento e o cotidiano de pessoas com diagnóstico do espectro esquizofrênico.

\section{Método}

A revisão aconteceu entre janeiro a abril de 2014 na Plataforma Bireme (BVS), escolhida por sua relevância na área acadêmica nacional. Esta plataforma agrega 12 bases de dados da área de saúde: LILACS, MEDLINE, ADOLEC, BBO, BDNENF, HISA, LEYES, MEDCARIB, REPIDISCA, OPAS, WHOLIS E DESASTRES. A cobertura inclui publicações do Brasil, Estados Unidos, Espanha, dentre outros países com ênfase nas áreas médica e biomédica, ciências da saúde, saúde pública, odontologia, enfermagem, legislação básica em saúde, engenharia básica e meio ambiente.

Foram utilizadas as seguintes palavras-chave: cotidiano, saúde mental, esquizofrenia, doença mental, psicose, vida diária, atividades cotidianas, daily life, everyday life, mental illness, schizophrenia, experience e narratives. Construíram-se 16 combinaçôes a partir delas: Cotidiano e Saúde Mental; Cotidiano e Doença Mental; Cotidiano e Psicose; Cotidiano e Esquizofrenia; Vida Diária e Saúde Mental; Vida Diária e Doença Mental; Vida Diária e Psicose; Vida Diária e Esquizofrenia; Atividades Cotidianas e Saúde Mental; Atividades Cotidianas e Psicose; Atividades Cotidianas e Esquizofrenia; Daily Life e Mental Illness e Narratives; Daily Life e Schizophrenia e Narratives; Daily Life e Mental Illness e Experience; Everyday Life 3 e Mental Illness; Everyday Life e Schizophrenia.

O primeiro acesso a BVS ocorreu a partir das palavras-chave acima citadas. Pelos títulos dos artigos encontrados, identificou-se quais palavras-chave e combinaçóes revelavam trabalhos pertinentes ao objeto do projeto de mestrado, no que diz respeito à relação entre esquizofrenia, cotidiano e experiência do adoecimento.

Foram selecionados os artigos que continham em seu título ao menos uma das palavras-chave, e posteriormente, através da leitura dos resumos, buscou-se aqueles que contemplavam ao menos duas temáticas do projeto (esquizofrenia, saúde mental, 
cotidiano, narrativas, experiência do adoecimento, serviços comunitários de saúde).

Destacam-se as seguintes combinaçôes na Tabela 1 a seguir com a quantidade de publicaçóes encontradas.

Dentre os primeiros 144 resumos selecionados, apenas 15 artigos foram lidos e analisados, por apresentarem entrevistas ou narrativas de pessoas com esquizofrenia relatando as próprias percepçóes sobre sua experiência de adoecimento e seu cotidiano. Segue-se Tabela 2 com as referências dos artigos lidos na íntegra.

A partir desse pequeno número de artigos encontrados, considerou-se ampliar a busca a novas bases de pesquisas e informaçôes. A hipótese foi de que outros campos, como a Sociologia e Antropologia, produzem pesquisas narrativas sobre a experiência do adoecimento a partir de outros pressupostos. Sendo assim, essa produçáo estaria indexada em outras Plataformas e Bases de Dados internacionais, multidisciplinares e de Ciências Sociais.

Foram selecionadas seis bases: Academic One File (Base de Dados Multidisciplinar - 1980; Academic Search Premier (Base de Dados Multidisciplinar - 1920 a 2014); Proquest Central (Plataforma Multidisciplinar - 1950 a 2014); Francis (Base de Dados Ciências Sociais - 1972 a 2014); Jstor (Base de Dados Ciências Sociais - 2000 a 2014); Web of Science (Base de Dados Multidisciplinar - 1945 a 2014). Os critérios de seleção para estas bases foram: assinatura Capes e/ou Unicamp; acervo com diferentes períodos de abrangência e de diferentes nacionalidades, site acessível na língua inglesa e suporte de descritores bilíngues.

Descreve-se na Tabela 3 a seguir as Bases de Dados selecionadas e a quantidade de publicaçóes encontradas seguindo os mesmos critérios de seleção dos artigos da busca na BVS.
Dentre os 137 resumos selecionados e descritos na tabela 3, 67 artigos apresentaram entrevistas ou narrativas de pessoas com esquizofrenia relatando as próprias percepções sobre a experiência de adoecimento, sendo esse número mais expressivo do que o encontrado na BVS (15).

Trabalhou-se com a leitura e análise dos 82 artigos (BVS +6 bases) e apresenta-se a seguir a discussão sobre os achados em relação à temática deste artigo.

\section{Resultados e Discussão}

O primeiro artigo sobre a temática pesquisada datava de 1959, embora os estudos sobre esquizofrenia e cotidiano continuem escassos até a década de 1990. A partir desta data, observa-se um crescimento de publicaçóes que se acelera nos primeiro anos da década de 2000. A palavra "cotidiano" manteve-se como sinônimo para um conjunto de AVDs nas produçôes internacionais. Considerando as sete bases de dados listadas nas Tabelas 1 e 3, 281 artigos foram selecionados, $90 \%$ destes de origem internacional e pouco menos de um terço (82 artigos) relatam/descrevem e valorizam as narrativas em primeira pessoa sobre a experiência de adoecimento.

Os dados apresentados acima demonstram que, no campo do conhecimento cientifico (BOURDIEU, 1983), ainda são majoritários os estudos que consideram os transtornos mentais a partir das variáveis como etiologia, diagnóstico, prognóstico, agudeza, cronicidade e curso da enfermidade (COELHO; ALMEIDA FILHO, 2002). Estudos acerca do contexto histórico-cultural e socioambiental em que a pessoa está inserida, de seu modo de ser-no-mundo, de se relacionar com os outros e de viver e compreender a própria experiência de adoecimento, permanecem escassos, embora seja sabido que fenômenos sociais e políticos afetam diretamente a condição de saúde das pessoas (SARACENO, 2001; SERPA JUNIOR et al., 2011).

Tabela 1. Palavras-chave e quantidade de Publicações encontradas.

\begin{tabular}{lccc}
\hline \multicolumn{1}{c}{ Combinação de Palavras-chave } & $\begin{array}{c}\mathbf{N}^{\mathbf{o}} \text { de Publicações } \\
\text { Encontradas }\end{array}$ & $\begin{array}{c}\mathbf{N}^{\mathbf{o}} \text { de Publicações } \\
\text { Selecionadas } \\
\text { Filtro por Título }\end{array}$ & $\begin{array}{c}\mathbf{N}^{\mathbf{o}} \text { de Publicações } \\
\text { Selecionadas }\end{array}$ \\
Filtro por Resumo
\end{tabular}

Fonte: Portal Regional da BVS (2014). 
Tabela 2. Artigos que apresentam entrevistas ou narrativas de pessoas com esquizofrenia relatando as próprias percepções sobre sua experiência de adoecimento e seu cotidiano.

BORG, M.; DAVIDSON, L. The nature of recovery as lived in everyday experience. Journal of Mental Health, Londres, v. 17, n. 2, p. 129-140, 2008. Disponível em: <http://informahealthcare.com/doi/abs/10.1080/096382 30701498382? journalCode $=$ jmh $>$. Acesso em: 20 nov. 2014

CORIN, E.; LAUZON, G. Positive withdrawal and the quest for meaning: the reconstruction of experience among schizophrenics. Psychiatry, Washington, v. 55, n. 3, p. 266-281, 1992. Disponível em: <http://pesquisa. bvsalud.org/portal/resource/pt/mdl-1509013>. Acesso em: 20 nov. 2014.

DAVIDSON, L. et al. Remission and recovery in Schizophrenia: practitioner and patient perspectives. Schizophrenia Bulletin, Oxford, v. 34, n. 1, p. 5-8, 2008. Disponível em: <http://pesquisa.bvsalud.org/portal/resource/pt/mdl17984297>. Acesso em: 26 nov. 2014.

DIAS, M. K. A experiência social da psicose no contexto de um Centro de Atenção Psicossocial. Ciência \& Saúde Coletiva, Rio de Janeiro, v. 16, n. 4, p. 2155-2164, 2011. Disponível em: <http://www.scielosp.org/pdf/ csc/v16n4/v16n4a15.pdf $>$. Acesso em: 25 nov. 2014.

FIGUEIRÓ, R. A.; DIMENSTEIN, M. O cotidiano de usuários de CAPS: empoderamento ou captura. Fractal: Revista de Psicologia, Niterói, v. 22, n. 2, p. 431-446, 2010. Disponível em: <http://pesquisa.bvsalud.org/portal/ resource/pt/lil-557898>. Acesso em: 20 nov. 2014.

JENKINS, J. H.; CARPENTER-SONG E. The new paradigm of recovery from schizophrenia: cultural conundrums of improvement without cure. Culture, Medicine and Psychiatry, Dordrecht, v. 29, n. 4, p. 379-413, 2005. Disponível em: <http://pesquisa.bvsalud.org/portal/resource/pt/mdl-16773457>. Acesso em: 22 nov. 2014.

LOPES, T. S. et al. O processo de restabelecimento na perspectiva de pessoas com diagnóstico de transtornos do espectro esquizofrênico e de psiquiatras na rede pública de atenção psicossocial. Saúde e Sociedade, São Paulo, v. 21, n. 3, p. 558-571, 2012. Disponível em: $<$ http://pesquisa.bvsalud.org/portal/resource/pt/lil-654480>. Acesso em: 06 nov. 2014.

MOLL, M. F.; SAEKI, T. A vida social de pessoas com diagnóstico de esquizofrenia usuárias de um Centro de Atenção Psicossocial. Revista Latino-Americana de Enfermagem, Ribeirão Preto, v. 17, n. 6, p. 995-1000, 2009. Disponível em: $<$ http://www.scielo.br/scielo.php?pid=S010411692009000600011\&script=sci_arttext $>$. Acesso em: 28 nov. 2014.

NASI, C.; SCHNEIDER, J. F. O Centro de Atenção Psicossocial no cotidiano dos seus usuários. Revista da Escola de Enfermagem da USP, São Paulo, v. 45, n. 5, p. 1157-1163, 2011. Disponível em: <http://www.scielo. br/pdf/reeusp/v45n5/v45n5a18.pdf >. Acesso em: 28 nov. 2014.

Utiliza o conceito de cotidiano de Alfred Schutz (1979).

SALLES, M. M.; BARROS, S. Reinternação em hospital psiquiátrico: a compreensão do processo saúde/doença na vivência do cotidiano. Revista da Escola de Enfermagem da USP, São Paulo, v. 41, n. 1, p. 73-81, 2007. Disponível em: <http://pesquisa.bvsalud.org/portal/resource/pt/lil-450461>. Acesso em: 20 nov. 2014.

Utiliza o conceito de cotidiano de Agnes Heller (1994).

SALLES, M. M.; BARROS, S. Relações do cotidiano: a pessoa com transtorno mental e sua rede de suporte social. Physis Revista de Saúde Coletiva, Rio de Janeiro, v. 21, n. 2, p. 561-579, 2011. Disponível em: <http:// pesquisa.bvsalud.org/portal/resource/pt/lil-596067>. Acesso em: 21 nov. 2014.

Utiliza o conceito de cotidiano de Agnes Heller (2004).

SALLES, M. M.; BARROS, S. Vida cotidiana após adoecimento mental: desafio para atenção em saúde mental. Acta Paulista de Enfermagem, São Paulo, v. 22, n. 1, p. 11-16, 2009. Disponível em: <http://www.scielo.br/ scielo.php?pid=S0103-21002009000100002\&script=sci_abstract $>$. Acesso em: 29 nov. 2014.

Utiliza o conceito de cotidiano de Agnes Heller (2004).

SANTOS, M. R. P.; NUNES, M. O. Território e saúde mental: um estudo sobre a experiência de usuários de um Centro de Atenção Psicossocial, Salvador, Bahia, Brasil. Interface - Comunicação, Saúde, Educação, Botucatu, v. 15 , n. 38 , p. 715-726, 2011. Disponível em: <http://pesquisa.bvsalud.org/portal/resource/pt/lil-602019>. Acesso em: 20 nov. 2014.

WAGNER, L. C. et al. Cuestiones existenciales em la esquizofrenia: percepción de portadores y cuidadores. Revista de Saúde Pública, São Paulo, v. 45, n. 2, p. 401-8, 2011. Disponível em: < <ttp://www.scielo.br/pdf/rsp/ v45n2/2299.pdf>. Acesso em: 20 nov. 2014.

YILMAZ, M. et al. Social processes of participation in everyday life among persons with schizophrenia. International Journal of Qualitative Studies on Health and Well-being, Londres, v. 4, n. 4, p. 267-279, 2009. Disponível em: <http://informahealthcare.com/doi/abs/10.3109/17482620903113112>. Acesso em: 29 nov. 2014. 
Tabela 3. Plataformas e Base de Dados/quantidade de Publicações encontradas.

\begin{tabular}{lccc}
\hline \multicolumn{1}{c}{ Plataformas e Base de Dados } & $\begin{array}{c}\mathbf{N}^{\mathbf{0}} \text { de Publicações } \\
\text { Encontradas }\end{array}$ & $\begin{array}{c}\mathbf{N}^{\mathbf{o}} \text { de Publicações } \\
\text { Selecionadas } \\
\text { Filtro Título }\end{array}$ & $\begin{array}{c}\mathbf{N}^{\circ} \text { de Publicações } \\
\text { Selecionadas } \\
\text { Filtro Resumo }\end{array}$ \\
\hline $\begin{array}{l}\text { 1. Academic One File (Base de Dados } \\
\text { Multidisciplinar - 1980 a 2014) }\end{array}$ & 112 & 36 & 15 \\
$\begin{array}{l}\text { 2. Academic Search Premier (Base de Dados } \\
\text { Multidisciplinar - 1920 a 2014) }\end{array}$ & 281 & 98 & 58 \\
$\begin{array}{l}\text { 3. Proquest Central (Plataforma } \\
\text { Multidisciplinar - 1950 a 2014) }\end{array}$ & 2.197 & 130 & 23 \\
$\begin{array}{l}\text { 4. Francis (Base de Dados Ciências Sociais - } \\
\text { 1972 a 2014) }\end{array}$ & 5.661 & 44 & 11 \\
$\begin{array}{l}\text { 5. Jstor (Base de Dados Ciências Sociais - } \\
\text { 2000 a 2014) }\end{array}$ & 1.020 & 31 & 13 \\
6. Web of Science (Base de Dados & 256 & 36 & 17 \\
Multidisciplinar - 1945 a 2014) & & & 137 \\
Total & 7.527 & 375 & \\
\hline
\end{tabular}

Fonte: Sistema de Bibliotecas da Unicamp (2015).

A partir de 1970, um crescente número de pesquisas sobre esquizofrenia e a vida cotidiana enfatizam a necessidade de compreender a vida diária das pessoas a partir de suas narrativas e experiências (DAVIDSON, 2002; BATES, 2004).

Alguns aspectos parecem determinar esse crescimento:

- o aparecimento de estudos longitudinais sobre a esquizofrenia, que datam da década de 1960, realizados em vários países, que mostram que há um amplo espectro de desfecho da esquizofrenia, mesmo quando ela se manifesta de modo severo e persistente, ligados ao contexto sociocultural;

- a consolidação de lutas e movimentos contra os manicômios, com propostas de novos modelos de cuidado, bem como a garantia de direitos sociais para seus egressos. Preconizados nos países de cultura anglo-saxã, ítalo-ibérica, com fortalecimento de projetos de cuidado em saúde mental como a desospitalização, desinstitucionalização e a reabilitação psicossocial (BARROS, 1994);

- surgimento de novas formas de investigar o processo saúde/doença/cuidado, fortalecendo pesquisas em que a própria pessoa avalia sua vida, narra o que considera importante para si, uma vez que há novas alternativas de tratamento, em liberdade, centradas na reabilitação psicossocial e em serviços territoriais (MOLL; SAEKI, 2009).
Isso não se restringiu à saúde mental. Na década de 1980, surgiram estudos etnográficos examinando a medicina e seus procedimentos como um sistema cultural, com especial atenção às relaçóes médico-paciente, medicalização da doença e a burocratização do atendimento. A produçáo no campo científico, todavia, ainda se mantém restrita sobre a experiência encarnada e seus relatos em forma de narrativa. Com o levantamento bibliográfico, isso se tornou evidente, pois a quantidade de artigos não selecionados entre o primeiro filtro (título) e o segundo filtro (resumo) revelou o forte investimento em pesquisas quantitativas, relacionadas à neurociência, efeitos dos medicamentos e escalas avaliativas de padrôes comportamentais esperados após diferentes tratamentos em saúde mental que não levam em conta a experiência da pessoa adoecida.

A maioria dos artigos encontrados e selecionados pelo resumo nesta revisão ainda reduziam o cotidiano às atividades de vida diária e tomavam como papel do profissional de saúde adequá-las a uma vida mais saudável. Indicavam atividades moralmente aceitas em determinadas culturas, mas não questionavam como essas pessoas estão vivendo, por vezes em espaços alternativos, por vezes já bem inseridas em outros contornos sociais e institucionais estabelecidos, como famílias, religiôes, tratamentos alternativos. Logo, o cotidiano reduzido às AVDs continua sendo utilizado de forma indiscriminada e descontextualizado historicamente, não incorporando a diversidade cultural e social (GALHEIGO, 2003).

Borg e Davidson (2008) afirmam que a recuperação da doença mental grave é frequentemente vista como um processo individual, que ocorre em contextos de tratamento pouco vinculados à cultura e às relaçóes 
sociais das pessoas. Contudo, pesquisas recentes começam a explorar a recuperação como um processo social e que se desenvolve na vida cotidiana, tanto na sua simplicidade como na complexidade das experiências diárias.

Lysaker e Lysaker (2010) também concordam que os estudos sobre a esquizofrenia tendem a examinar a vida de pessoas pelos impactos biológicos e sociais em processos individuais e descritos por profissionais da saúde. No entanto, afirmam que pesquisas que deixam de considerar as dimensóes em primeira pessoa sobre a doença podem ser incompletas. Defendem pesquisas longitudinais explorando as possíveis implicações no tratamento e valorizando a dimensáo do adoecimento em primeira pessoa para alcançar descriçóes mais completas sobre essa experiência e formas de recuperação.

As afirmaçóes de Lysaker e Lysaker (2010), Borg e Davidson (2008) conferem com o encontrado neste levantamento. A maioria das pesquisas valorizaram métodos focados no discurso em terceira pessoa. Mesmo as metodologias de narração em primeira pessoa por vezes traziam a análise do cotidiano de forma a reduzi-lo às atividades de vida diária, por vezes padronizadas (testes de qualidade de vida, comportamental e funcionalidade), não valorizando o processo das pessoas, táo pouco a singularidade da experiência do adoecimento e como isso impacta o cotidiano.

Logo, podemos perceber que diferentes autores - Slade, Davdison, Moll, Lysaker -, de diferentes culturas e nacionalidades, defendem as pesquisas qualitativas, com métodos adequados para a construção de narrativas em primeira pessoa sobre a experiência de adoecimento, para assim compreender os processos saúde/doença/cuidado de forma complexa, a fim de contribuir de modo consistente com formas de cuidado e na construção de serviços assistenciais que valorizem o cotidiano singular de seus usuários.

Para Brown (1995), estudar como a doença é socialmente construída, como as forças sociais moldam a compreensão e ações na saúde, explorando os efeitos de classe, raça, sexo, língua, tecnologia, cultura, da economia política e institucional, das estruturas profissionais e das normas na formação de conhecimento, se faz necessário para entender as suposiçóes sobre a prevalência, incidência, tratamento e significado da doença. Assim, alguns estudiosos procuraram descobrir as interaçóes do cotidiano e das doenças a partir da experiência das pessoas. Começam investigando as atividades sociais (organizaçôes, instituições) e construçôes da experiência da doença; decisóes em relação ao tratamento; compreensôes do que constitui resultados. Contudo, a descoberta de um diagnóstico é frequentemente carregada de disputa, o que proporciona muitos conflitos sociais em torno de questóes da medicina e da saúde (BROWN, 1995).

O diagnóstico de esquizofrenia tem especial impacto no funcionamento social e na vida cotidiana das pessoas. No entanto, há poucas pesquisas a respeito do que as pessoas fazem no seu contexto social e o que caracteriza suas participaçóes nos processos sociais. A Reabilitação Psicossocial preocupa-se com três eixos centrais à vida das pessoas: o eixo morar, o eixo trocar identidades e o eixo produzir e trocar mercadorias e valores (SARACENO, 2001). Alguns paradigmas, como da recuperação centrada no cliente em terapia ocupacional, destacam a importância das relaçôes de apoio e terapêuticas, incluindo confiança, o respeito pelas escolhas e prioridades do cliente, escuta ativa e empatia (MÂNGIA, 2002; YILMAZ et al., 2009).

Alguns estudos antropológicos que discutiam sintomas presentes na esquizofrenia foram identificados. Nestes trabalhos, com indígenas australianos, os pesquisadores Suryania, Welchb e Cox (2013) viram que os participantes consideravam as vozes significativas e positivas, por vezes forneciam orientaçôes sobre questôes da vida cotidiana e as relacionavam com a presença de espíritos ancestrais. Neste grupo indígena, a presença de vozes não foi considerada ruim e estigmatizante. Isto indica que, ao trabalhar com pessoas que vivenciam alucinações auditivas, é importante reconhecer a presença das vozes na vida das pessoas, e como estas impactam a vida em relação à autoestima, aos comportamentos $\mathrm{e}$ às relaçóes interpessoais (SURYANIA; WELCHB; COX, 2013).

Apesar de os autores acima citados focarem em um sintoma específico, não exclusivo e determinante para o diagnóstico de esquizofrenia, revelam que a construção cultural define nomes diferentes e formas distintas de lidar com certos fenômenos corporais. Porém, qualquer que seja o fenômeno, ele deve ser considerado na sua dimensão cotidiana, na experiência vivida de cada sujeito em seu contexto sociocultural.

Um dos pesquisadores mais citados e com mais artigos encontrados nas bases de dados acessadas é o pesquisador Larry Davidson, professor de Psicologia do Departamento de Psiquiatria na Universidade de Yale. Suas pesquisas concentram-se na interface recovery/recuperação e saúde mental, investigando processos de recuperaçáo na psicose, usando o apoio dos pares ${ }^{4}$ e outras estratégias de engajamento social, o desenvolvimento de métodos de pesquisa qualitativa e participativa e intervençôes psicossociais de base comunitária. 
Davidson et al. (1997) e Davidson (2002) trabalhou com o conceito de cotidiano através de dois autores: Schutz (1972) e Gullestad (1989 apud DAVIDSON et al., 1997). Schutz (1972) conceitua cotidiano como "o mundo da vida" com base na fenomenologia, sendo um dos autores citados nos artigos desse levantamento, e Gullestad (1989 apud DAVIDSON et al., 1997), seguidora de Schutz, considera que a vida cotidiana pode ser invisível por ser muito visível. Gullestad sugere duas perspectivas sobre a vida cotidiana: 1 . Organizacional/funcional. 2. Vivida/Subjetiva, ou na fenomenologia denominada "o mundo da vida", com foco na experiência significativa do ser humano.

Nos estudos de Borg e Davidson (2008), o tratamento e o processo de recovery estáo nas trivialidades da vida cotidiana, que náo pode ser considerara como trivial, porque os problemas práticos decorrentes $\mathrm{da}$ doença mental afetam a vida cotidiana.

No Brasil, os primeiros artigos brasileiros (SALLES; BARROS, 2009; MOLL; SAEKI, 2009; NASI; SCHINEIDER, 2011) que estudaram a experiência do adoecimento em esquizofrenia e os impactos no cotidiano datam do início do século XXI e tratam o conceito de cotidiano através de A. Heller (2004) e também A. Schutz (1979). Salles e Barros (2009) optam por fundamentar-se em Agnes Heller, pois esta autora realiza uma análise crítica do real, oferecendo alicerce para o conhecimento da atividade prática social dos sujeitos históricos concretos. Já Nasi e Schineider (2011) buscam na sociologia fenomenológica de Alfred Schutz a base teórica para sua pesquisa.

Poucos autores nacionais e internacionais explicitam a definição de cotidiano. A maioria não descreveu nos artigos como concebem essa noção, mas nas referências bibliográficas dos artigos encontram-se pistas a partir dos autores utilizados. Além de Schutz e Heller, os mais citados nas bibliografias, há referências a De Certeau, Lefebvre e Maffesoli.

A menor quantidade de trabalhos qualitativos e de narrativas que abordam o conceito de cotidiano associado ao campo da saúde mental e da experiência de adoecimento em esquizofrenia pode encontrar argumentos explicativos em Costa (2005). Esta autora afirma que o cotidiano pode ser fluido e de difícil definição, talvez por isso existam poucos trabalhados sobre o cotidiano na interface saúde mental/sociologia, sendo na sua grande maioria textos descritivos das atividades diárias, sem profundidade nas singularidades das relaçôes afetivas, sociais, econômicas, dentre tantas outras que não podemos captar.
Costa (2005), estudando Deleuze e Guattari, reflete sobre o cotidiano com a diluição de fronteiras e barreiras, a ausência de demarcações sociais, a crescente mobilidade de tudo e de todos, a desterritorialização, o nomadismo, os "não-lugares" do homem contemporâneo. Ele é anônimo, mas tem referências globais (mesmas marcas de bebida, de comida, roupas). Mesmo assim, pode encontrar nesse "não-lugar" a possibilidade de geração de espaços alternativos de vida, com características próprias, com relaçóes sociais ricas e experiências complexas.

Além disso, a própria experiência de adoecimento mental pode mudar profundamente percepçóes de si, do mundo e dos outros. Contudo, as pessoas com uma doença mental, como qualquer outro cidadão, buscam significado na vida. Assim, os serviços de saúde mental devem apoiar soluçôes cotidianas para todos os problemas diários das pessoas (SLADE, 2012).

Portanto, estudar o cotidiano de pessoas com diagnóstico do espectro esquizofrênico na contemporaneidade torna-se um desafio ainda maior e mais complexo devido à nossa cultura em constante transformaçáo e com fronteiras táo tênues como as da razão/ilusão.

A estruturação dos serviços e seus profissionais precisam se preparar para acolher, entender e colaborar para a solução dos problemas e desafios cotidianos contemporâneos complexos e em constante transformaçáo apresentados pelas pessoas com esquizofrenia, bem como valorizar as "pequenas" conquistas diárias, que comportam um mundo de significaçóes e ajudam a fortalecer o sentido da vida para todos.

Campos (2009, p. 69) mostra que a tarefa dos serviços de saúde não é apenas atender à doença; o que significa que cabe aos profissionais de saúde não só a capacidade de ajudar as pessoas a combater doenças, mas também ajudá-las a se transformarem, de forma que a doença, mesmo podendo ser um limite, não as impeça de vivenciar novas possibilidades na vida, combinando a racionalidade clínico/sanitária e os interesses/desejos dos usuários, “[...] que depende da adoção do compartilhamento da construção de uma narrativa e de sua interpretação entre o trabalhador/ especialista e o sujeito/usuário".

Logo, há a necessidade de os serviços territoriais em saúde mental organizarem-se para a continuidade do cuidado de longo prazo para as pessoas que experienciam o adoecimento, haja vista a relevância e necessidade do cuidado, mas também pela necessidade de especial atenção para as mudanças que os ciclos da vida impóem ao cotidiano dessas pessoas. 
Os cuidados em saúde mental não podem focar apenas nos treinos e avaliação de habilidades sociais e de desempenho, mas na criação e manutenção de espaços de ser, estar e habitar (SARACENO, 2001), como atividades sociais, de trabalho e de lazer significativas para as pessoas. A Reabilitação Psicossocial se propóe a refletir e atuar neste complexo conjunto de lugares, de organizaçóes de serviços, de intervençóes em estruturas sanitárias e sociais do território, configurando um processo que implica a abertura de espaços de negociaçáo para o usuário, com a família, com a comunidade circundante e com os serviços que se ocupam do usuário e da ampliação do seu poder contratual (SARACENO, 2001).

Assim, reconhecendo a importância da experiência significativa na vida cotidiana e seus diversos componentes, o maior desafio encontra-se em explicitar, capturando e reconhecendo a simplicidade do cotidiano, a complexidade da vida cotidiana entre as pessoas com experiência de adoecimento mental (BORG; DAVIDSON, 2008).

\section{Conclusão}

A partir desta revisão narrativa, percebe-se a pertinência e responsabilidade no cenário contemporâneo das pesquisas/investigação em saúde mental centradas no vivido pelos usuários em tratamento sob o novo modelo de atenção, buscando diálogo não só entre os iguais, mas sim com as singularidades, ouvindo e respeitando as diferentes experiências de adoecimento, buscando valorizar a dimensão subjetiva e empoderando as pessoas com transtorno mental grave, no contexto sociocultural brasileiro.

O número de publicaçôes de estudos qualitativos que valorizam a experiência narrada das pessoas que vivenciam a esquizofrenia é crescente, mas ainda bem inferior, comparativamente, ao número de estudos quantitativos que valorizam os aspectos biológicos, centrados nos efeitos dos medicamentos, ou que avaliem as mudanças comportamentais de uma perspectiva externa àquele que vive o adoecimento. O conceito de cotidiano também se apresenta como desafio às pesquisas devido à sua complexidade e forte tendência dos autores em reduzi-lo ou entendê-lo como sinônimo das AVDs.

Refletir sobre o processo de adoecimento em esquizofrenia na sociedade e seus impactos na vida cotidiana das pessoas que o vivenciam (estigma, preconceito, invalidaçáo) se faz necessário. $\mathrm{O}$ reconhecimento do cotidiano vivido em sua complexidade/totalidade, a partir de estudos de narrativas sobre a experiência de adoecimento, contribui para que o cuidado prestado às pessoas com esquizofrenia em serviços territoriais de saúde tipo CAPS, bem como em outros espaços sociais, leve em conta o ponto de vista de quem vive o processo de adoecimento e suas necessidades, fortalecendo as Políticas Públicas de Saúde Mental e a Reforma Psiquiátrica Brasileira.

A vida cotidiana se retroalimenta na vida social. Pode-se não ter os mesmos gestos, ações e formas de realizar as atividades, mas isso deve ser entendido não como excludente de possibilidades e potências, mas sim como fenômenos singulares, onde caminhar com a diferença pode revelar novos entendimentos das experiências de adoecimento e contribuir para a construção de um tratamento que dialogue com as reais necessidades dos usuários. A Terapia Ocupacional, como um núcleo de saber voltado/centrado na açáo cotidiana das pessoas que acompanha, tem como compromisso desenvolver estudos que considerem o cotidiano a partir da experiência vivida, buscando assim colaborar para a construção de relaçôes terapêuticas dialéticas dos processos saúde/doença/cuidado, como também com novas pesquisas no campo abordado por este artigo.

\section{Referências}

BARROS, D. D. Jardins de Abel: desconstrução do manicômio de Trieste. São Paulo: Lemos/EDUSP, 1994.

BATES, J. A. Use of narrative interviewing in everyday information. Library \& Information Science Research, Washington, v. 26, n. 1, p. 15-28, 2004. Disponível em: <http://www.sciencedirect.com/science/article/pii/ S0740818803000938>. Acesso em: 25 nov. 2014.

BORG, M.; DAVIDSON, L. The nature of recovery as lived in everyday experience. Journal of Mental Health, Londres, v. 17, n. 2, p. 129-140, 2008. Disponível em: <http://informahealthcare.com/doi/abs/10.1080/09638 230701498382 ?journalCode $=\mathrm{jmh}>$. Acesso em: 20 nov. 2014.

BOURDIEU, P. O campo científico. In: ORTIZ, R. (Org.). Pierre Bourdieu. São Paulo: Ática, 1983. p. 122 155.

BROWN, P. Naming and Framing: The Social Construction of Diagnosis and Illness. Journal of Health and Social Behavior, Washington, v. 35, p. 34-52, 1995. Número Especial. Disponível em: <http://www.jstor.org/discover/10.2307/2626956? uid=2\&uid=4\&s $\mathrm{id}=21105268415383>$. Acesso em: 25 nov. 2014 .

CAMPOS, G. W. S. Clínica e Saúde Coletiva compartilhadas: teoria Paidéia e reformulação ampliada do trabalho em saúde. In: CAMPOS, G. W. S. et al. (Org.). 
Tratado de Saúde Coletiva. São Paulo: Hucitec, 2009. p. 41-80.

COELHO, M. T. A. D.; ALMEIDA FILHO, N. Conceitos de saúde em discursos contemporâneos de referência científica. História, Ciências, Saúde-Manguinhos, Rio de Janeiro, v. 9, n. 2, p. 315-33, 2002. Disponível em: <http://www.scielo.br/pdf/hcsm/v9n2/a05v9n2. pdf $>$. Acesso em: 25 nov. 2014.

CORIN, E.; LAUZON, G. Positive withdrawal and the quest for meaning: the reconstruction of experience among schizophrenics. Psychiatry, Washington, v. 55, n. 3, p. 266-281, 1992. Disponível em: <http://pesquisa.bvsalud.org/portal/resource/pt/mdl-1509013>. Acesso em: 20 nov. 2014.

COSTA, A. M. N. O cotidiano nos múltiplos espaços contemporâneos. Psicologia: Teoria e Pesquisa, Brasília, v. 21, n. 3, p. 365-373, 2005. Disponível em:<http://www. scielo.br/pdf/ptp/v21n3/a14v21n3>. Acesso em: 25 nov. 2014.

DANTAS, C. R.; ODA, A. M. G. R. Cartografia das pesquisas avaliativas de serviços de saúde mental no Brasil (2004-2013). Physis Revista de Saúde Coletiva, Rio de Janeiro, v. 4, n. 24, p. 1127-1179, 2014. Disponível em: <http://www.scielo.br/scielo.php?pid=S0103$-73312014000401127 \&$ script=sci_arttext $>$. Acesso em: 26 nov. 2014.

DAVIDSON, L. et al. Phenomenological and participatory research on Schizophrenia: recovering the person in theory and practice. Journal of Social Issues, Washington, v. 53, n. 4, p. 767-784, 1997. Disponível em: <http://onlinelibrary.wiley.com/doi/10.1111/j.1540-4560.1997. tb02460.x/abstract>. Acesso em: 26 nov. 2014.

DAVIDSON, L. et al. Remission and recovery in Schizophrenia: practitioner and patient perspectives. Schizophrenia Bulletin, Oxford, v. 34, n. 1, p. 5-8, 2008. Disponível em: <http://pesquisa.bvsalud.org/portal/ resource/pt/mdl-17984297>. Acesso em: 20 nov. 2014.

DAVIDSON, L. Intentionality, identity, and delusions of control in Schizophrenia: a Husserlian perspective. Journal of Phenomenological Psychology, Leiden, v. 33, n. 1, p. 39-58, 2002. http://dx.doi. org/10.1163/156916202320900419.

DIAS, M. K. A experiência social da psicose no contexto de um Centro de Atenção Psicossocial. Ciência \& Saúde Coletiva, Rio de Janeiro, v. 16, n. 4, p. 2155-2164, 2011. Disponível em: <http://www.scielosp.org/pdf/csc/ v16n4/v16n4a15.pdf>. Acesso em: 25 nov. 2014.

FIGUEIRÓ, R. A.; DIMENSTEIN, M. O cotidiano de usuários de CAPS: empoderamento ou captura. Fractal: Revista de Psicologia, Niterói, v. 22, n. 2, p. 431-446, 2010. Disponível em: <http://pesquisa.bvsalud.org/portal/resource/pt/lil-557898>. Acesso em: 20 nov. 2014.

GALHEIGO, S. M. O cotidiano na terapia ocupacional: cultura, subjetividade e contexto histórico-social. Revista de Terapia Ocupacional da Universidade de São Paulo,
São Paulo, v. 14, n. 3, p. 104-109, 2003. Disponível em: <http://www.revistas.usp.br/rto/article/view/13924/0>. Acesso em: 26 nov. 2014.

HELLER, A. Sociologia de la vida cotidiana. Barcelona: Península, 1994.

HELLER, A. O cotidiano e a história. São Paulo: Paz e Terra, 2004.

JENKINS, J. H.; CARPENTER-SONG, E. The new paradigm of recovery from schizophrenia: cultural conundrums of improvement without cure. Culture, $\mathrm{Me}$ dicine and Psychiatry, Dordrecht, v. 29, n. 4, p. 379-413, 2005. Disponível em: <http://pesquisa.bvsalud.org/ portal/resource/pt/mdl-16773457>. Acesso em: 22 nov. 2014.

LOPES, T. S. et al. O processo de restabelecimento na perspectiva de pessoas com diagnóstico de transtornos do espectro esquizofrênico e de psiquiatras na rede pública de atenção psicossocial. Saúde e Sociedade, São Paulo, v. 21, n. 3, p. 558-571, 2012. Disponível em: <http:// pesquisa.bvsalud.org/portal/resource/pt/lil-654480>. Acesso em: 06 nov. 2014.

LYSAKER, P. H.; LYSAKER, J. T. Schizophrenia and alterations in self-experience: a comparison of 6 perspectives. Schizophrenia Bulletin, Oxford, v. 36, n. 2, p. 331 340, 2010. Disponível em: <http://schizophreniabulletin.oxfordjournals.org/content/36/2/331.short>. Acesso em: 26 nov. 2014.

MÂNGIA, E. F. Contribuiçôes da abordagem canadense "prática de Terapia Ocupacional centrada no cliente" e dos autores da desinstitucionalização italiana para a terapia ocupacional em saúde mental. Revista de Terapia Ocupacional da Universidade de São Paulo, São Paulo, v. 13, n. 3, p. 127-134, 2002. Disponível em: <http:// www.revistas.usp.br/rto/article/view/13907/0>. Acesso em: 28 nov. 2014.

MINAYO, M. C. S. O desafio do conhecimento. São Paulo: Hucitec, 2012.

MOLL, M. F.; SAEKI, T. A vida social de pessoas com diagnóstico de esquizofrenia usuárias de um Centro de Atenção Psicossocial. Revista Latino-Americana de Enfermagem, Ribeirão Preto, v. 17, n. 6, p. 995-1000, 2009. Disponível em: <http://www.scielo.br/scielo. php?pid=S0104-11692009000600011\&script=sciartte xt>. Acesso em: 28 nov. 2014.

NASI, C.; SCHNEIDER, J. F. O Centro de Atenção Psicossocial no cotidiano dos seus usuários. Revista $d a$ Escola de Enfermagem da USP, São Paulo, v. 45, n. 5, p. 1157-1163, 2011. Disponível em: <http://www.scielo. br/pdf/reeusp/v45n5/v45n5a18.pdf>. Acesso em: 28 nov. 2014.

PORTAL REGIONAL DA BVS. São Paulo: BIREME, 2014. Disponível em: <http://www.bireme.br/php/index.php>. Acesso em: 27 fev. 2014.

ROTHER, E. T. Revisão sistemática X Revisão narrativa. Acta Paulista de Enfermagem, São Paulo, v. 20, n. 2, p. v-vi, 2007. Disponível em: <http://www.redalyc.org/articulo.oa?id=307026613004>. Acesso em: 28 nov. 2014 . 
SALLES, M. M.; BARROS, S. Reinternação em hospital psiquiátrico: a compreensão do processo saúde/ doença na vivência do cotidiano. Revista da Escola de Enfermagem da USP, São Paulo, v. 41, n. 1, p. 73-81, 2007. Disponível em: <http://pesquisa.bvsalud.org/portal/resource/pt/lil-450461>. Acesso em: 20 nov. 2014.

SALLES, M. M.; BARROS, S. Vida cotidiana após adoecimento mental: desafio para atenção em saúde mental. Acta Paulista de Enfermagem, São Paulo, v. 22, n. 1, p. 1116, 2009. Disponível em: <http://www.scielo.br/scielo. php?pid=S0103-21002009000100002\&script=sci_abstract>. Acesso em: 29 nov. 2014.

SALLES, M. M.; BARROS, S. Relaçóes do cotidiano: a pessoa com transtorno mental e sua rede de suporte social. Physis Revista de Saúde Coletiva, Rio de Janeiro, v. 21, n. 2, p. 561-579, 2011. Disponível em: <http:// pesquisa.bvsalud.org/portal/resource/pt/lil-596067>. Acesso em: 21 nov. 2014.

SALLES, M. M.; MATSUKURA, T. S. Estudo de revisão sistemática sobre o uso do conceito de cotidiano no campo da terapia ocupacional no Brasil. Cadernos de Terapia Ocupacional da UFSCar, São Carlos, v. 21, n. 2, p. 265-273, 2013. http://dx.doi.org/10.4322/ cto. $2013.028 .</$ jrn $>$.

SANTOS, M. R. P.; NUNES, M. O. Território e saúde mental: um estudo sobre a experiência de usuários de um Centro de Atenção Psicossocial, Salvador, Bahia, Brasil. Interface - Comunicação, Saúde, Educação, Botucatu, v. 15, n. 38, p. 715-726, 2011. Disponível em: <http:// pesquisa.bvsalud.org/portal/resource/pt/lil-602019>. Acesso em: 20 nov. 2014.

SARACENO, B. Libertando identidades: da reabilitação psicossocial à cidadania possível. Rio de Janeiro: TeCorál Instituto Franco Basaglia, 2001.

SCHUTZ, A. Fenomenologia del mundo social. Introducción a La sociologia comprensiva. Buenos Aires: Paidos, 1972.
SCHUTZ, A. Fenomenologia e relaçóes sociais. Rio de Janeiro: Zahar, 1979.

SERPA JUNIOR, O. D. et al. Experiência, narrativa e conhecimento: a perspectiva do psiquiatra e a do usuário. Edital MCT/CNPq/CT-Saúde/MS/SCTIE/DECIT, n. 33/2008. Rio de Janeiro: Laboratório de Estudos e Pesquisas em Psicopatologia e Subjetividade, UFRJ, 2011. (Relatório Técnico Final).

SISTEMA DE BIBLIOTECAS DA UNICAMP - SBU. Campinas: Unicamp, 2015. Disponível em: <http:// www.sbu.unicamp.br>. Acesso em: 29 mar. 2014.

SLADE, M. Everyday solutions for everyday problems: how mental health systems can support recovery. Psychiatric Services, Washington, v. 63, n. 7, p. 702-704, 2012. Disponível em: <http://ps.psychiatryonline.org/ doi/abs/10.1176/appi.ps.201100521>. Acesso em: 29 nov. 2014

SURYANIA, S.; WELCHB, A.; COX, L. The phenomena of auditory hallucination as described by Indonesian people living with Schizophrenia. Archives of Psychiatric Nursing, Washington, v. 27, n. 6, p. 312-318, 2013. Disponível em: <http://www.sciencedirect.com/ science/article/pii/S0883941713000939>. Acesso em: 29 nov. 2014

WAGNER, L. C. et al. Cuestiones existenciales em la esquizofrenia: percepción de portadores y cuidadores. Revista Saúde Pública, São Paulo, v. 45, n. 2, p. 401 8, 2011. Disponível em: <http://www.scielo.br/pdf/rsp/ v45n2/2299.pdf>. Acesso em: 20 nov. 2014.

YILMAZ, M. et al. Social processes of participation in everyday life among persons with schizophrenia. International Journal of Qualitative Studies on Health and Well-being, Londres, v. 4, n. 4, p. 267-279, 2009. Disponível em: <http://informahealthcare.com/doi/ abs/10.3109/17482620903113112>. Acesso em: 29 nov. 2014.

\section{Contribuição dos Autores}

Ellen Cristina Ricci foi responsável pela concepção, organização de fontes e análises, redação e revisão do texto. Erotildes Maria Leal realizou a concepção, redação e revisão do texto. Ambas autoras aprovaram a versão final do texto.

\section{Notas}

${ }^{1}$ Este material é parte da pesquisa de mestrado da primeira autora do artigo, aprovado pelo Comitê de Ética da Faculdade de Ciências Médicas, número 870/2009 - Adendo 24/06/2014.

${ }^{2}$ Daily Life é o termo que se aproxima de "atividade de vida diária” na tradução em português.

${ }^{3}$ Everydaylife é o termo usado como conceito de "cotidiano" na língua inglesa.

${ }^{4}$ Apoio dos Pares: termo utilizado para designar a participação de pessoas que passam ou passaram pelo processo de adoecimento em pesquisas acadêmicas não apenas como sujeitos pesquisados, mas como pesquisadores. 